\title{
The CAD of Group Animation based on Firefly Algorithm and Ant Colony Algorithm Fusion Algorithm
}

\author{
Xu Shijie,Jiang Shuming,Zhang Jianfeng Wei Zhiqiang, Li Jian,Wang Shuai \\ The Information Research Institute of Shandong Academy of Sciences \\ Jinan, China
}

\begin{abstract}
Firefly Algorithm (FA) is a new meta-heuristic evolutionary algorithm and it has fast calculation and excellent global search capability. Ant Colony Algorithm (ACA) has a positive feedback mechanism and distributed optimization method. Its solution has high accuracy. For characteristics of FA and ACA, the FA and ACA fusion algorithm has advantages of two algorithms to overcome their own disadvantages. In a typical function test, it shows good optimization performance and efficient time performance. And then FA and ACA fusion algorithm is applied to group animation path design to save the animation designer's labor, and it provides a new design method for the computer animation production.
\end{abstract}

Keywords-Firefly Algorithm (FA), Fusion Algorithm, Path Routing, Computer Animation, CAD

\section{INTRODUCTION}

Firefly Algorithm is a new optimization algorithm In evolutionary computation with the characters of simple, less parameters, fast calculation, strong global search capability, easy to implement and so on, it can be used to solve a multiobjective optimization problem [1,2]. In recent years, Such algorithms have been improved and applied In a wide range of engineering projects[3,4], further evidence of its superiority of solving optimization problems have been proved, such as function optimization, underground pipe network design, traveling salesman problem[5,6]. From the current Research situation at home and abroad, computeraided animation based on evolutionary computation has become a hot topic currently, There are many experts and scholars carried out research from different sides of the field[7]. The fusion algorithm pre-use Firefly algorithm to generate a global optimal solution group, post-use the ant colony algorithm to produce a fast and accurate global optimal solution path. Through the algorithm performance testing and the application of group animation path routing, it has achieved a good performance of path optimization.

\section{FIREFLY ALGORITHM}

\section{A. Firefly Algorithm}

The flashing light of fireflies is an amazing sight in the summer sky in the tropical and temperate regions. There are about two thousand firefly species, and most fireflies produce short and rhythmic flashes. The pattern of flashes is often unique for a particular species. The flashing light is produced by a process of bioluminescence, and the true functions of such signaling systems are still debating.
However, two fundamental functions of such flashes are to attract mating partners (communication), and to attract potential prey. In addition, flashing may also serve as a protective warning mechanism. The rhythmic flash, the rate of flashing and the amount of time form part of the signal system that brings both sexes together. Females respond to a male's unique pattern of flashing in the same species, while in some species such as photuris, female fireflies can mimic the mating flashing pattern of other species so as to lure and eat the male fireflies who may mistake the flashes as a potential suitable mate. We know that the light intensity at a particular distance $r$ from the light source obeys the inverse square law. That is to say, the light intensity I decreases as the distance $r$ increases in terms of $\mathrm{I} \propto 1 / \mathrm{r} 2$. Furthermore, the air absorbs light which becomes weaker and weaker as the distance increases. These two combined factors make most fireflies visible only to a limited distance, usually several hundred meters at night, which is usually good enough for fireflies to communicate [8]. The flashing light can be formulated in such a way that it is associated with the objective function to be optimized, which makes it possible to formulate new optimization algorithms.

Firefly algorithm is a heuristic swarm intelligence computing technology. It is idealized by three rules:

1) all the fireflies will be attracted regardless of their gender.

2) The brightness of their attractiveness is directly proportional.

3) The brightness of fireflies influence and determine objective function. For the maximization problem, the brightness can be simply proportional to the value of objective function.

\section{B. The Algorithm Process and Parameters}

\section{The Algorithm Process}

Firstly Define object function $\mathrm{f}(\mathrm{x})$.

Secondly Generate initial population of fireflies $i(i=1$,

$2, \ldots, n)$, define initial light intensity I0 .

Thirdly define the light absorption $\gamma$.

Fourthly iteration calculation:

While $(\mathrm{t}<$ MaxGeneration)

For $\mathrm{i}=1: \mathrm{n}$ all $\mathrm{n}$ fireflies

For $\mathrm{j}=1$ : $\mathrm{i}$ all $\mathrm{n}$ fireflies

If (Ij > Ii), Move firefly End if

Attractiveness varies with distance

Evaluate new solutions update light intensity

End for $\mathrm{j}$ End for $\mathrm{i}$

Rank the fireflies and find the current best 
End while

Fifthly process and output the global optimization solution.

2. The main parameters and expressions Attractive Expression:

$$
\beta(r)=\beta_{0} e^{-r^{r^{2}}}
$$

Move Expression:

$$
x_{i}=x_{i}+\beta_{0} e^{-\gamma^{r_{i}^{2}}}\left(x_{j}-x_{i}\right)+\alpha\left(\text { rand }-\frac{1}{2}\right)
$$

\section{ANT COLONy ALGORITHM}

\section{A. Ant Colony Algorithm}

Ant colony algorithm is inspired by the real ants from the nest to find the shortest path to food, it is used to solve the complex optimization problems. Ant evolution works through the optimum solution in the role of selfenhancement, it solves the problem of global optimal solution in the direction of evolution, and ultimately to obtain the optimal solution.

Optimization objective function is assumed:

$$
\min Z=f(x) \quad x \in[a, b]
$$

Initial time the amount of pheromone on a variety of paths are equal, let $\tau_{i j}(0)=\mathrm{c}$ (c is a constant), ant $\mathrm{k}(\mathrm{k}=$ $1,2, \ldots, \mathrm{m}), \mathrm{m}$ is the total number of ants in the colony, $p_{i j}^{k}(t)$ shows the probability of ant $\mathrm{k}$ in position $\mathrm{i}$ transfers to the position $\mathrm{j}$ in time $\mathrm{t}$ :

$$
p_{i j}^{k}(t)=\left\{\begin{array}{l}
\frac{\left[\tau_{i j}(t)\right]^{\alpha}\left[\eta_{i j}(t)\right]^{\beta}}{\sum_{s \in \text { allowed }_{k}}\left[\tau_{i s}(t)\right]^{\alpha}\left[\eta_{i s}(t)\right]^{\beta}}, j \in \text { allowed }_{k} \\
0 \quad, j \notin \text { allowed }_{k}
\end{array}\right.
$$

$\tau_{i j}(t)$ indicates the pheromone intensity on the path ij at time $\mathrm{t}, \eta_{i j}$ indicates the inspired information, $\alpha$ indicates the weight of the pheromone, $\beta$ indicates the weight of heuristic information.

After Ant to complete a cycle, the amount of information on the path to update and adjust according to the following formula:

$$
\begin{gathered}
\tau_{i j}(t+n)=(1-\rho) \tau_{i j}(t)+\Delta \tau_{i j}, \\
\Delta \tau_{i j}=\sum_{k=1}^{m} \Delta \tau_{i j}^{k}
\end{gathered}
$$

$\rho$ represents the pheromone evaporation coefficient, 1$\rho$ indicates pheromone retention rate, $\Delta \tau_{i j}^{k}$ indicates the ant $\mathrm{k}$ ant to stay in this loop of information on the incremental path ij.

Different pheromone update strategy, Dorigo. M made the basic ant colony algorithm in three different models, such as Ant-Cycle model, the overall use of information:
$\Delta \tau_{i j}^{k}=\left\{\begin{array}{l}\frac{Q}{L_{k}}, \text { in this circle ant kpass by path ij } \\ 0, \quad \text { or }\end{array}\right.$

Q represents the pheromone intensity. $L_{k}$ the total path length of the ant $\mathrm{k}$ in this cycle by taking the circle walk , and it is the objective function value.

\section{B. Algorithm parameters}

Objective function: $\min Z=f(x) x \in[a, b]$ the Initial time the amount of pheromone is equal on a variety of paths, $\mathrm{m}$ is the total number of ant, the probability of transferring formula:

$$
p_{i j}^{k}(t)=\left\{\begin{aligned}
\frac{\left[\tau_{i j}(t)\right]^{\alpha}\left[\eta_{i j}(t)\right]^{\beta}}{\sum_{s \in a l l o w_{e} d_{k}}\left[\tau_{i s}(t)\right]^{\alpha}\left[\eta_{i s}(t)\right]^{\beta}}, j \in \text { allowed } \\
0 \quad, j \notin \text { allowed }{ }_{k}
\end{aligned}\right.
$$

the amount of information update formula: $\tau_{i j}(t+n)=(1-\rho) \tau_{i j}(t)+\Delta \tau_{i j}, \Delta \tau_{i j}=\sum_{k=1}^{m} \Delta \tau_{i j}^{k}$

According to the different pheromone update strategies, Dorigo. $\mathrm{M}$ presented the basic ant colony algorithm in three different models, such as this algorithm uses Ant-Cycle model, the overall use of pheromone is the objective function value.

\section{THE BASIC FRAMEWORK OF THE ANT COLONY AND FIREFLY FUSION ALGORITHM}

A. Basic Framework:

The basic framework, shown in Figure 1:

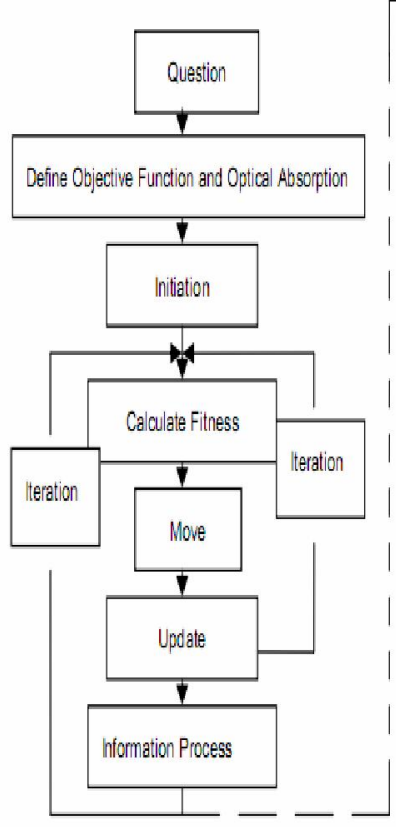

FA Module

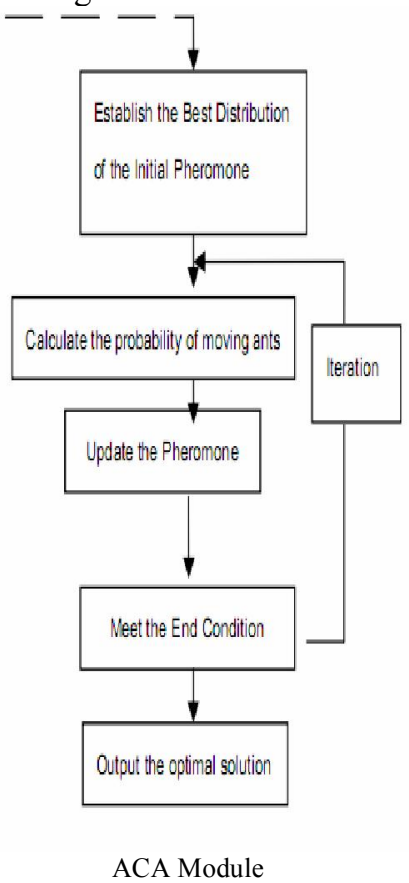

ACA Module
Figure 1. the basic framework of the fusion algorithm

\section{B. Algorithm}

1) Firefly module: consult the title II.B the first step to the fifth step。

2) Ant colony algorithm module: 
The first step the preliminary mixing iterative process produces the best solution at this time.

The second step the ants move according to the ant pheromone intensity and heuristic function by the probability of moving within the global scope.

The third step the global pheromone update.

The fourth step if it meets the end conditions, the algorithm calculates the objective function value of the results. Otherwise, back to the second step.

The fifth step it determines the optimal solution.

\section{Performance Of The Algorithm And Its APPLICATION}

\section{A. the algorithm test}

To test the efficiency of the algorithm, by Using the following test function to test:

1.Sphere function:

$$
\begin{gathered}
f_{1}(x)=\sum_{i=1}^{n} x_{i}{ }^{2} \\
-5.12 \leq x_{i} \leq 5.12
\end{gathered}
$$

min-value 0 , optimization position $(0,0, \ldots 0)$

2.Rosenbrock:

$$
\begin{gathered}
f_{2}(x)=\sum_{i=1}^{n}\left[100\left(x_{i+1}-x_{i}^{2}\right)^{2}+\left(x_{i}-1\right)^{2}\right] \\
-5 \leq x_{i} \leq 5
\end{gathered}
$$

min-value 0 , optimization position $(1,1, \ldots 1)$

3.Griewank:

$$
\begin{gathered}
f_{3}(x)=\frac{1}{4000} \sum_{i=1}^{10} x_{i}^{2}-\prod_{i=1}^{10} \cos \left(\frac{x_{i}}{\sqrt{i}}\right)+1 \\
-600 \leq x_{i} \leq 600
\end{gathered}
$$

min-value 0 , optimization position $(0,0, \ldots 0)$

4.Schaffer's:

$$
\begin{gathered}
f_{4}(x)=\sum_{i=1}^{N-1}\left(x_{i}^{2}+x_{i+1}^{2}\right)^{0.25}\left[\sin ^{2}\left(\left(x_{i}^{2}+x_{i+1}^{2}\right)^{0.1}\right)+1\right] \\
-100 \leq x_{i} \leq 100
\end{gathered}
$$

min-value 0 , optimization position $(0,0, \ldots 0)$

Performance test in the algorithm was used the proposed algorithm parameters In reference[1]. Particle number 100, the number of iterations is 500. The algorithm operates independently 50 times, the average optimization as results, and the experimental results shown in Table 1:

B. Application

Group animation path is the groups form a continuous trajectory on the screen, the basic requirements are: 1 animation path diversity. 2 It is a kind of dispersion and aggregation purposes of continuous movement. Group animation path is formed by the individual animation path. Particles by creating firefly and its trajectory as a potential solution, a large number of particles through communicating with each other, exchanging information, form the changing trajectory, animation path diversity provides practical basis for the animation designer to choose. Based on the above study, the firefly and ant colony fusion algorithm simulate the animation path planning in fish foraging behavior as an example: Figure 2 shows the initial state of the random behavior. Figure 4 shows the cluster feeding behavior. Set and change parameters, it will produce different types of paths, resulting in a number of renderings, it allows the simulation of group behavior in nature and making the group animation path planning quicker and easier, and also it provides designers a convenient tool.

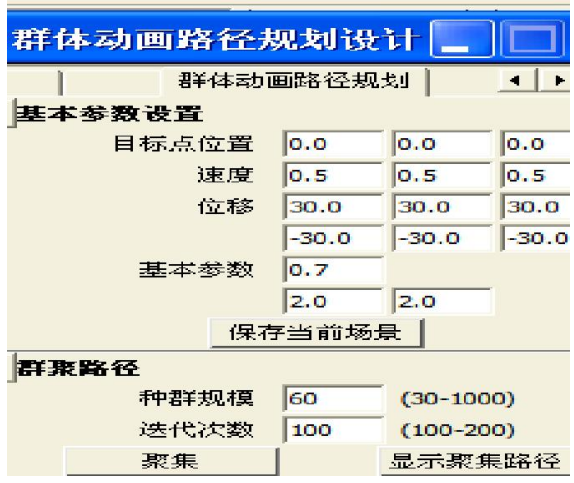

Figure 2. Initiation Parameters

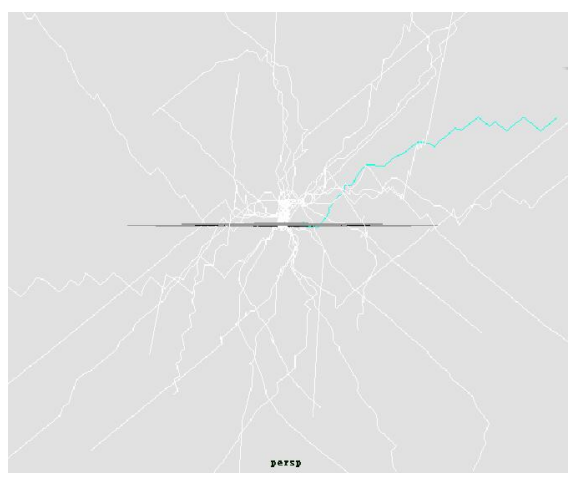

Figure 3. Path Diagram

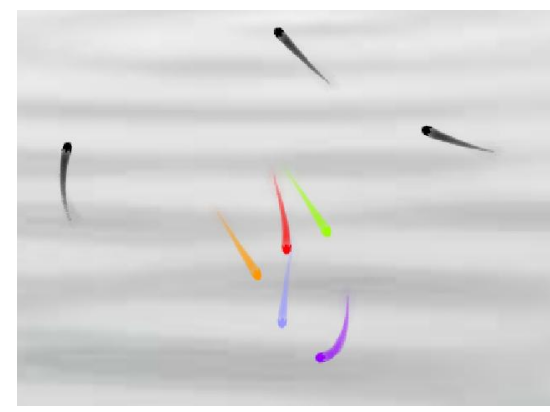

Figure 4. Example applications (Initiation)

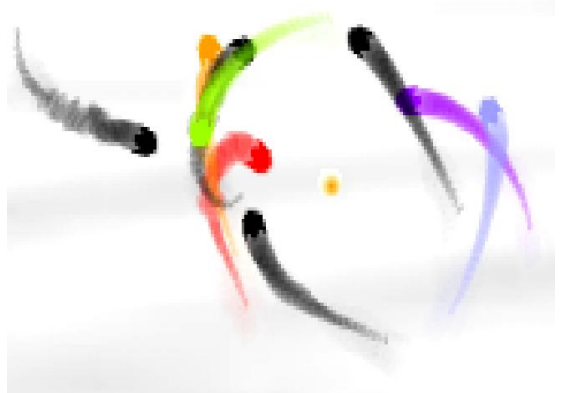

Figure 5. Example applications(group collective behavior) 


\section{SUMMARY}

Create particles, the fusion algorithm uses optimization ability of FA and the accurate solution ability of ACA and then put it in the algorithm test of continuous function, it shows that the algorithm has a good searching efficiency. The fusion algorithm provides a practical algorithm tool to promote the production of computer animation.

The basic theory research of fusion algorithm and its parameter selection, etc. need a lot of improvement. Although computer aided animation design is gradually rising, but many issues involved, such as the comparison of the evolutionary algorithm and its application in computeraided animation design, etc. the further study has a long way to go.

\section{REFERENCES}

[1] Elbctagi E,Hegazy T,Grierson D.Comparison among five evolutionary-based optimization algorithms[J].Advanced engineering Informatics,2005,19(1):43-53
[2] Elbehairy H,Elbeltagi E,Hegazy T,et ul.Comparison of teo evolutionary algorithms for optimization of bridge deck repairss[J].Computer-aided Civil and Infrastructure Engineering,2006,21:561-572.

[3] Shie Y H,Atiquzzaman M.Optimal design of water distrubution network using shuffled complex evolution[J].The Institution of Engineers,2004,44(1):93-107.

[4] Krishnanand, K. N., and Ghose, D. (2005). 'Detection of multiple source locations using a glowworm metaphor with applications to collective robotics,' Swarm Intelligence Symposium, 2005. SIS 2005. Proceedings 2005 IEEE :84-91.

[5] Yang, X.S.: Nature-Inspired Metaheuristic Algorithms. Luniver Press (2008) :130-135.

[6] Luk Yu Qian, Zhang Songmao. From Story to Animation - the entire process of automatic generation of computer-assisted animation [J], Automation Technology, 2002,28 (3): 321-348.

[7] Zhang Shujun, Ban Xiao-Juan, Chen Yong, Chen Ge. Based on artificial intelligence and artificial life in computer animation [J], Computer Science, 2007,34 (10): 233-237.

[8] Xin-She Yang. Firefly Algorithms for Multimodal Optimization [C], O. Watanabe and T. Zeugmann (Eds.): SAGA 2009, LNCS 5792, pp. 169-178, 2009.

TABLE I. COMPARISON RESULTS OF THE ALGORITHM

\begin{tabular}{|c|c|c|c|c|c|c|c|c|}
\hline Function & \multicolumn{2}{|c|}{ Sphere } & \multicolumn{2}{c|}{ Rosenbrock } & \multicolumn{2}{c|}{ Griewank } & \multicolumn{2}{c|}{ Schaffer's } \\
\hline Algorithm & FA & FA-ACA & FA & FA-ACA & FA & FA & FA-ACA \\
\hline $\begin{array}{c}\text { Optimal } \\
\text { value }\end{array}$ & $2.177 \mathrm{E}-4$ & $0.088 \mathrm{E}-4$ & 1.864 & 1.673 & $6.015 \mathrm{E}-2$ & $4.231 \mathrm{E}-2$ & $5.496 \mathrm{E}-1$ & $5.054 \mathrm{E}-1$ \\
\hline $\begin{array}{c}\text { Average } \\
\text { Optimal } \\
\text { Value }\end{array}$ & $1.236 \mathrm{E}-3$ & $0.134 \mathrm{E}-3$ & 2.547 & 2.250 & $2.710 \mathrm{E}-1$ & $2.319 \mathrm{E}-1$ & 4.984 & 4.764 \\
\hline $\begin{array}{c}\text { Best } \\
\text { Solutions }\end{array}$ & 0 & 0 & 0 & 0 & 0 & 0 & 0 & 0 \\
\hline
\end{tabular}

